TORRES JLR; ARAÚJO AS; BARRETO AC; SILVA NETO OF; SILVA VR; VIEIRA DMS. 2015. Desenvolvimento e produtividade de couve-flor e repolho influenciados por tipos de cobertura do solo. Horticultura Brasileira 33: 510-514. DOI - http://dx.doi.org/10.1590/S0102-053620150000400017

\title{
Desenvolvimento e produtividade de couve-flor e repolho influenciados por tipos de cobertura do solo
}

\author{
José LR Torres; Adriano S Araújo; Antônio C Barreto; Onésio F Silva Neto; Venâncio R Silva; Dinamar \\ MS Vieira
}

Instituto Federal do Triângulo Mineiro, Uberaba-MG, Brasil; jlrtorres@iftm.edu.br; adriano.silva1994@hotmail.com; barreto@iftm.edu. br; onesioneto@gmail.com; venanciorodrigues1994@hotmail.com; marcinha_0202@hotmail.com

\section{RESUMO}

A utilização de plantas de cobertura antecedendo o cultivo de hortaliças tem sido uma das alternativas utilizadas para diminuir o uso de adubação mineral. Neste estudo avaliaram-se coberturas do solo aplicadas antes do plantio e seus efeitos em algumas características agronômicas e na produtividade de couve-flor e repolho. Utilizou-se o delineamento de blocos ao acaso, em esquema fatorial, com quatro coberturas do solo (crotalária juncea, braquiária, milheto e pousio) e duas culturas (couve-flor e repolho), com quatro repetições, em parcelas de $20 \mathrm{~m}^{2}(4 \times 5 \mathrm{~m})$. Foi determinado o número de folhas, altura, diâmetro da cabeça, do caule e horizontal, massa fresca e seca. As coberturas utilizadas influenciaram positivamente as características agronômicas da couve-flor e do repolho para diâmetro da cabeça $(55,1$ e 194,8 cm), diâmetro do caule (70,4 e 49,2 mm) e diâmetro horizontal (169,9 e 14,8 mm), massa fresca $(1,0$ e $2,8 \mathrm{~kg})$ e massa seca da cabeça $(73,4$ e $0,65 \mathrm{~g})$ e produtividade $(7,3$ e 19,2 t/ha) das culturas, respectivamente, quando cultivadas sobre os resíduos de braquiária. A massa fresca comercial de repolho $(2,8 \mathrm{~kg})$ foi superior ao padrão exigido pelo mercado consumidor brasileiro.

Palavras-chave: Brassica oleracea var. botrytis, Brassica oleracea var. capitata, plantas de cobertura, ciclagem de nutrientes, produtividade.

\begin{abstract}
Development and productivity of cauliflower and cabbage influenced by soil mulching types

The use of cover crops preceding vegetable cultivation has been one option to reduce the necessity of mineral fertilizers. In this study we evaluated the soil covers applied preceding planting and its effects on some agronomic characteristics and productivity of cauliflower and cabbage. We used a factorial randomized blocks design, with four soil covers (sunnhemp, brachiaria, millet and fallow) and two crops (cauliflower and cabbage) with four replications in plots of 20 $\mathrm{m}^{2}(4 \times 5 \mathrm{~m})$. We determined the number of leaves, height, head and stem diameter, and fresh and dry mass. Mulches influenced positively the agronomic characteristics of cauliflower and cabbage for head diameter (55.1 and $194.8 \mathrm{~cm})$, stem diameter $(70.4$ and $49.2 \mathrm{~mm})$ and horizontal diameter (169.9 and $148 \mathrm{~mm})$, fresh mass (1.0 to $2.8 \mathrm{~kg}$ ) and dry mass of the head (73.4 and $0.65 \mathrm{~g})$ and productivity (7.3 to $19.2 \mathrm{t} / \mathrm{ha}$ ) of the crops, respectively, when grown on brachiaria waste. The marketable fresh mass of cabbage $(2.8 \mathrm{~kg})$ was higher than the standard required by the Brazilian consumer market.
\end{abstract}

Keywords: Brassica oleracea var. botrytis, Brassica oleracea var. capitata, mulching crops, nutrient cycling, productivity.

(Recebido para publicação em 20 de maio de 2014; aceito em 7 de abril de 2015) (Received on May 20, 2014; accepted on April 7, 2015)

E ntre as várias hortaliças ofertadas aos consumidores brasileiros, a couve-flor e o repolho estão incluídos entre as três mais consumidas no país (Carvalho et al., 2013). Essas brássicas com alto valor nutritivo e comercial apresentam elevada capacidade de extração de nutrientes do solo (Silva et al., 2012), utilizando grande quantidade de fertilizantes no seu cultivo, o que aumenta o custo de produção (Oliveira et al., 2005).

Uma das alternativas utilizadas para diminuir o consumo de fertilizantes é o cultivo de plantas de cobertura antecedendo o plantio dessas hortaliças. Após serem manejadas, seus resíduos são depositados sobre a superfície do solo, promovendo a elevação do teor de matéria orgânica (MO) e da capacidade de troca catiônica (CTC) no solo causando maior disponibilidade de nutrientes (Leite et al., 2010) com a sua decomposição, além de manter a umidade do solo e controlar plantas invasoras (Espíndola et al., 2006). Esses efeitos são bastante variáveis, pois dependem da espécie utilizada, manejo dado à biomassa, época de semeadura, persistência dos resíduos sobre o solo, condições locais e da interação entre esses fatores (Teixeira et al., 2012).

Estudos têm mostrado que braquiária, crotalária, milheto e pousio (vegetação espontânea) aportam quantidades consideráveis de nutrientes ao solo, após o manejo das coberturas (Carvalho et al., 2011; Pacheco et al., 2011; Chioderoli et al., 2012; Teixeira et al., 2012; Assis 
et al., 2013). Avaliando o acúmulo e ciclagem de N, P, K, Ca e Mg nos resíduos de braquiária, crotalária, milheto e pousio na região do Triângulo Mineiro, Torres et al. (2008) e Torres \& Pereira (2008) observaram o acúmulo de 130,8; 13,$3 ; 214,7 ; 18,7$ e $21,1 \mathrm{~kg} / \mathrm{ha} ; 118,1$; 10,$8 ; 59,2 ; 42,6$ e $12,8 \mathrm{~kg} / \mathrm{ha} ; 165,5$; 22,$6 ; 218,9 ; 41,1$ e $22,6 \mathrm{~kg} /$ ha e 46,7 ; 3,$5 ; 23,8 ; 19,1$ e $6,3 \mathrm{~kg} / \mathrm{ha}$, enquanto que Fabian (2009) constatou o acúmulo de 81,$9 ; 6,4 ; 97,9 ; 20,9$ e $14,0 \mathrm{~kg} / \mathrm{ha}$; 90,$1 ; 2,1 ; 61,6 ; 29,5$ e $9,8 \mathrm{~kg} / \mathrm{ha} ; 66,5$; 22,$8 ; 117,5 ; 12,4$ e $8,3 \mathrm{~kg} / \mathrm{ha} ; 34,1 ; 2,1$; 46,$0 ; 9,4$ e 4,6 kg/ha para estas culturas respectivamente, sendo que nestes estudos a maior liberação destes nutrientes ocorreu até os 42 dias após o manejo.

Outra opção de aporte de nutrientes é o uso de adubação orgânica, via esterco animal ou vermicompostagem, pois essas culturas apresentam boa resposta à adubação orgânica (Fontanétti et al., 2006), mas os nutrientes disponibilizados pela matéria orgânica ocorrem de forma gradual e variam de acordo com a cultivar e a fonte de adubo utilizada (Brito \& Santos, 2010). Ricci et al. (1995) observaram que a adubação com composto orgânico tradicional (esterco) e vermicomposto para produção de alface proporcionou teores de $\mathrm{P}, \mathrm{Ca}, \mathrm{Mg}$ e $\mathrm{S}$ iguais à testemunha com adubação mineral. Diniz et al. (2007) constataram que a aplicação de 8,6 t/ha de biomassa seca de mucuna cinza, somados a 12,0 t/ha de composto orgânico causou o aumento do teor de $\mathrm{N}$ mineral no solo, que refletiu na maior produção do brócolis.

Para o repolho, Fontanétti et al. (2006) utilizaram 12,7 t/ha de biomassa seca de crotalária juncea, 8,50 t/ha de mucuna preta, 7,51 t/ha de feijão de porco e 5,2 t/ha de vegetação espontânea (pousio), que resultaram na produção de cabeças de brócolis com 1,4, 1,2, 1,2 e $2,0 \mathrm{~kg}$, respectivamente. Vargas et al. (2011), utilizando 50\% da dose recomendada de adubação mineral somado à biomassa seca de crotalária e feijão de porco, obtiveram produção de repolho similar à obtida com 100\% adubação mineral, com rendimento variando entre 1,9 e $2,3 \mathrm{~kg}$ por planta, que corresponde à produtividade de 47,0 a 58,0 t/ha.

Nesse estudo objetivou-se avaliar o uso de quatro coberturas do solo ante- cedendo o plantio e seus efeitos em algumas características agronômicas e no rendimento da couve-flor e do repolho.

\section{MATERIAL E MÉTODOS}

O estudo foi realizado na área experimental do Instituto Federal do Triângulo Mineiro (IFTM), Campus Uberaba-MG, entre dezembro/2012 e setembro/2013. O clima da região é classificado como Aw tropical quente, segundo Koppen, com inverno frio e seco. Na região ocorrem médias anuais de precipitação de $1600 \mathrm{~mm} /$ ano e temperatura de $22,6^{\circ} \mathrm{C}$ (Uberaba em dados, 2009). Entretanto, no período observou-se a ocorrência de precipitação nos meses de abril (167,7 mm), maio (97,9 mm), junho $(37,1 \mathrm{~mm})$ e julho (14,8 mm) e diminuição entre novembro (190,4 mm) e dezembro (224,7 mm), o que não é comum na região.

O solo da área foi classificado como Latossolo Vermelho distrófico de textura média (Embrapa, 2006), apresentando na camada arável: $\mathrm{pH} \mathrm{CaCl}_{2}=5,5 ; 76$ $\mathrm{mg} / \mathrm{dm}^{3}$ de $\mathrm{P}$ (resina); $2 \mathrm{mmol} / \mathrm{dm}^{3}$ de $\mathrm{K}^{+} ; 22 \mathrm{mmol} / \mathrm{dm}^{3}$ de $\mathrm{Ca}^{2+} ; 10 \mathrm{mmol} /$ $\mathrm{dm}^{3}$ de $\mathrm{Mg}^{2+} ; 17 \mathrm{mmol}_{\mathrm{c}} / \mathrm{dm}^{3}$ de $\mathrm{H}+\mathrm{Al} \mathrm{e}$ $19 \mathrm{~g} / \mathrm{dm}^{3}$ de matéria orgânica.

O delineamento utilizado foi de blocos ao acaso, em esquema fatorial (4x2), quatro tipos de cobertura: crotalária juncea (Crotalaria juncea); braquiária (Urochloa brizantha cv marandú), milheto ADR 500 (Pennisetum glaucum) e pousio (vegetação espontânea com predomínio de poáceas), duas culturas (couve-flor cv. Sharon e repolho cv. Astrus plus), 4 repetições. As parcelas possuíam área de $20 \mathrm{~m}^{2}$ (4x5 m).

Nessa área foi cultivada a soja sobre as mesmas coberturas do solo no ano de 2011, que foi colhida em março de 2012. No mês de dezembro de 2012, braquiária, crotalária e milheto foram semeadas com espaçamento de 45 $\mathrm{cm}$ entre as linhas, com 50, 25 e 50 sementes por metro, respectivamente. As coberturas foram dessecadas em março de 2013, quando mais de 50\% das plantas atingiram o máximo florescimento, aplicando-se a dose de $1440 \mathrm{~g} /$ ha de glifosato $+600 \mathrm{~g} /$ ha de paraquat. Para avaliação da biomassa seca (BS), coletou-se amostras do material vegetal em área de $2 \mathrm{~m}^{2}$ por parcela, sendo secas a $65^{\circ} \mathrm{C}$ por 72 horas e pesadas.

As mudas das hortaliças foram produzidas em casa de vegetação coberta, em bandejas de isopor de 128 células contendo o substrato comercial Plantmax ${ }^{\circledR}$. Antes do transplantio das mudas, as coberturas foram roçadas com roçadeira costal rente ao solo para que houvesse o acamamento da vegetação sobre a superfície do solo. As mudas foram transplantadas sobre esta palha no início de abril de 2013 no espaçamento de $80 \times 40 \mathrm{~cm}$. Não foram confeccionados canteiros na área. Foram consideradas as três linhas úteis centrais para avaliação das culturas.

As plantas foram irrigadas diariamente por aspersão, mantendo a umidade do solo próximo à capacidade de campo e as plantas infestantes foram controladas com capinas manuais. O sistema de irrigação utilizado foi aspersão convencional fixo, equipado com aspersores setoriais com vazão de $560 \mathrm{~L} / \mathrm{h}$ espaçados de 9 metros um do outro, com um tempo de irrigação de aproximadamente 20 minutos.

$\mathrm{Na}$ preparação das covas antes do transplantio das mudas foi utilizada a metade da adubação recomendada com composto orgânico (esterco bovino curtido) na dosagem de $10 \mathrm{t} /$ ha. No plantio foi utilizada a metade da adubação mineral recomendada para as culturas com base na análise do solo e de acordo com a recomendação da Comissão de Fertilidade do Solo do Estado de Minas Gerais (1999). Para a couve-flor e repolho utilizou-se $50 \mathrm{~kg} / \mathrm{ha}$ de $\mathrm{P}_{2} \mathrm{O}_{5}$, $50 \mathrm{~kg} / \mathrm{ha}$ de $\mathrm{k}_{2} \mathrm{O}$ e $75 \mathrm{~kg} / \mathrm{ha}$ de $\mathrm{N}$, sendo este ultimo parcelado no plantio, 30 e 45 dias após, além de $1 \mathrm{~g}$ de ácido bórico (17,5\% de B) por cova.

A colheita da couve-flor foi realizada à medida que as inflorescências apresentavam desenvolvimento completo com botões florais ainda unidos, cabeças compactas e firmes. Com início aos 90 dias após semeadura, a colheita se estendeu por mais 30 dias, período este onde foram realizadas avaliações a cada três dias. Após a colheita, as plantas foram levadas ao laboratório para avaliações do número de folhas (NF), altura (A), diâmetro da cabeça (Dcab), do caule 
(Dcau) e horizontal (DH), massa fresca (MFC) e massa seca (MSC) da cabeça e produtividade (Prod).

Para o repolho, a colheita foi realizada quando a compacidade (firmeza) das cabeças alcançava a aceitação comercial, que teve inicio aos 100 dias após a semeadura e se estendeu por mais 20 dias. Foi determinado o número de folhas (NF), altura (A), diâmetro da cabeça (Dcab), do caule (Dcau) e horizontal (DH) com auxilio de um paquímetro, massa fresca (MF) e seca (MS) da cabeça e produtividade (Prod).

Os valores das características avaliadas foram submetidos à análise de variância, utilizando-se o programa estatístico SISVAR. Aplicou-se o teste F para significância e as médias foram comparadas pelo teste LSD-student a $5 \%$ de probabilidade.

\section{RESULTADOS E DISCUSSÃO}

Para as coberturas vegetais avaliadas, observou-se que a produção de biomassa seca $(\mathrm{BS})$ foi superior na área com milheto $(8,6 \mathrm{t} / \mathrm{ha})$ e pousio $(8,6 \mathrm{t}$ ) ha), que diferiram das outras coberturas (Tabela 1). Comparativamente estes valores são considerados baixos para milheto e elevados para o pousio, pois essas coberturas têm apresentado produção de BS acima de 10,0 t/ha e abaixo de 6,0 t/ha para estas culturas, respectivamente, em outros estudos conduzidos na mesma região e período de avaliação (Torres et al, 2008; Pacheco et al., 2011; Assis et al., 2013). Esses valores podem ser justificados pelo elevado volume de precipitação que ocorreu no período em que as plantas foram cultivadas (dezembro de 2011 a março de 2012), pois essas plantas têm sistema radicular fasciculado, que exploram maior área lateral e em profundidade, absorvendo maiores quantidades de água e nutrientes.

Para a crotalária, a produção de biomassa seca foi baixa (4,6 t/ha) comparada aos dados de $12,7 \mathrm{t} /$ ha obtidos por Fontanétti et al. (2006) em Lavras-MG e 58,7 t/ha observados por Vargas et al. (2011) em Viçosa-MG. Entretanto, foram similares aos observados por Torres et al. $(2005,2008)$ e Fabian (2009) que variaram entre 4,0 e 7,0 t/ha em estudos conduzidos nos últimos dez anos com plantas de cobertura na mesma região e condições edafoclimáticas. A utilização de diferentes variedades de crotalaria ( $C$. juncea, $C$. spectabilis e C. ochroleuca) e a baixa qualidade da semente disponível na região justificam as diferenças observadas entre os estudos.

Em couve-flor ocorreram diferenças significativas para as características agronômicas avaliadas, sendo que os melhores resultados para diâmetro da cabeça (Dcab) $(55,1$ e 54,7 cm), horizontal (DH) (169,9 e 175,5 mm), massa fresca $(\mathrm{MFC})(1,0$ e 1,2 $\mathrm{kg})$ e massa seca (MSC) da cabeça (73,4 e 74,1 g) e produtividade (Prod) (7,3 e 8,2 t/ha) foram observados quando a cultura foi cultivada sobre os resíduos culturais de braquiária e crotalária (Tabela 2). Entretanto, todos os valores observados no presente estudo, com exceção do diâmetro médio da cabeça (Dcab), foram inferiores aos obtidos por Morais Junior et al. (2012) para a cultivar Sharon, que atingiu $1,5 \mathrm{~kg}$ de massa média da cabeça e $30,7 \mathrm{t} /$ ha de produtividade.

O melhor rendimento da couve-flor cultivada sobre os resíduos de crotalária e braquiária pode ser justificado pelo desempenho destas coberturas quando cultivadas no Cerrado. Torres et al. (2005), Boer et al. (2008), Crusciol \& Soratto (2009), Marouelli et al. (2010), Pacheco et al. (2011), Vargas et al. (2011) e Assis et al. (2013) destacam que milheto, braquiária e crotalária têm sido as principais espécies utilizadas como coberturas de solo no Cerrado, pois estas plantas caracterizam-se pela elevada produção de biomassa, alta capacidade de extração de nutrientes do solo, com amplas vantagens de ciclagem de nutrientes, principalmente $\mathrm{N}$ e $\mathrm{K}$, reduzindo os riscos de perdas por lixiviação.

Fontanétti et al. (2006), em Lavras-MG, observaram que a crotalária foi a cobertura com maior e o pousio com a menor absorção e acúmulo de nutrientes em sua biomassa, sendo liberados quando os resíduos dessas culturas entraram em processo de decomposição. Torres et al. (2008) e Fabian (2009), em Uberaba-MG, observaram que a decomposição dos resíduos de crotalária e braquiária ocorre de forma acelerada, quando comparada ao milheto, sendo que a maior ciclagem de nutrientes ocorreu até 42 dias após o manejo das coberturas.

Com relação ao diâmetro médio da cabeça, cultivares com cabeça de maior tamanho são mais valorizadas no mercado, pois se enquadram nas classes superiores das normas de classificação para padrões de comercialização (Hortbrasil, 2011). Contudo, tem-se verificado diferentes magnitudes para esta característica, menores ou maiores para esta mesma cultivar (Monteiro et al., 2010).

Em repolho verificou-se que o número de folhas, diâmetro da cabeça, do caule, horizontal e produtividade foram superiores quando a cultura foi cultivada sobre resíduos culturais de braquiária

Tabela 1. Produção de biomassa verde (BV), seca (BS) e teor de biomassa seca (TBS) das coberturas do solo que antecederam o cultivo das hortaliças, em 2013 \{production of fresh (BV) and dried biomass (BS) and dry biomass content (TBS) of soil mulching preceding the cultivation of vegetables in 2013 . Uberaba, IFTM, 2013.

\begin{tabular}{|c|c|c|c|}
\hline \multirow{2}{*}{ Coberturas } & BV & BS & \multirow{2}{*}{ TBS (\%) } \\
\hline & \multicolumn{2}{|c|}{ (t/ha) } & \\
\hline Braquiária & $37,3 \mathrm{~b}$ & $6,5 b$ & $17,9 \mathrm{~b}$ \\
\hline Crotalária & $36,5 \mathrm{c}$ & $4,6 \mathrm{c}$ & $12,7 \mathrm{c}$ \\
\hline Milheto & $40,7 \mathrm{a}$ & $8,6 \mathrm{a}$ & $21,2 \mathrm{a}$ \\
\hline Pousio & $41,3 \mathrm{a}$ & $8,6 \mathrm{a}$ & $20,9 \mathrm{a}$ \\
\hline $\mathrm{F}$ & $1,811 *$ & $2,007^{*}$ & $0,258 *$ \\
\hline $\mathrm{CV}(\%)$ & 13,54 & 14,85 & 9,82 \\
\hline DMS & 0,7 & 1,79 & 2,50 \\
\hline
\end{tabular}

*Significativo $(\mathrm{p}<0,05)$. Médias seguidas de mesma letra na coluna não diferem entre si pelo teste LSD-student $(\mathrm{p}<0,05)$ \{ ${ }^{*}$ significant $(\mathrm{p}<0.05)$. Means followed by the same letter in the column do not differ by LSD-student $(\mathrm{p}<0.05)\}$. 
Tabela 2. Características agronômicas da couve-flor sobre resíduos de diferentes coberturas do solo \{agronomic characteristics of cauliflower cultivated with different plant residues used as soil mulching\}. Uberaba, IFTM, 2013.

\begin{tabular}{|c|c|c|c|c|c|c|c|c|}
\hline \multirow{2}{*}{ Coberturas } & \multirow{2}{*}{ NF } & Altura & Dcab & \multirow{2}{*}{$\begin{array}{l}\text { Dcau } \\
(\mathrm{mm})\end{array}$} & \multirow{2}{*}{ DH } & \multirow{2}{*}{$\begin{array}{c}\text { MFC } \\
\text { (kg) }\end{array}$} & \multirow{2}{*}{$\begin{array}{c}\text { MSC } \\
\text { (g) }\end{array}$} & \multirow{2}{*}{$\begin{array}{l}\text { Prod } \\
\text { (t/ha) }\end{array}$} \\
\hline & & \multicolumn{2}{|c|}{$(\mathbf{c m})$} & & & & & \\
\hline Braquiária & 24 & $11,4 \mathrm{a}$ & $55,1 \mathrm{a}$ & $70,4 \mathrm{a}$ & 169,9 a & $1,0 \mathrm{a}$ & $73,4 \mathrm{a}$ & $7,3 \mathrm{a}$ \\
\hline Crotalária & 23 & $10,9 \mathrm{~b}$ & $54,7 \mathrm{a}$ & $30,3 \mathrm{c}$ & $175,5 \mathrm{a}$ & $1,2 \mathrm{a}$ & $74,1 \mathrm{a}$ & $8,2 \mathrm{a}$ \\
\hline Milheto & 24 & $10,7 \mathrm{~b}$ & $51,7 \mathrm{~b}$ & $45,9 \mathrm{~b}$ & $157,6 \mathrm{~b}$ & $0,9 \mathrm{~b}$ & $60,4 \mathrm{~b}$ & $5,9 \mathrm{~b}$ \\
\hline Pousio & 24 & $10,2 \mathrm{c}$ & $48,5 \mathrm{c}$ & $47,5 \mathrm{~b}$ & $153,1 \mathrm{~b}$ & $0,8 \mathrm{~b}$ & $54,6 \mathrm{~b}$ & $5,3 \mathrm{~b}$ \\
\hline $\mathrm{F}$ & $0,258^{\text {ns }}$ & $2,448^{*}$ & $3,410^{*}$ & $2,192 *$ & $2,834^{*}$ & $2,395^{*}$ & $1,741^{*}$ & $2,392 *$ \\
\hline CV (\%) & 3,82 & 5,58 & 5,48 & 19,04 & 6,56 & 22,00 & 19,40 & 22,01 \\
\hline DMS & 1,71 & 0,43 & 2,42 & 12,47 & 15,17 & 0,05 & 12,97 & 1,76 \\
\hline
\end{tabular}

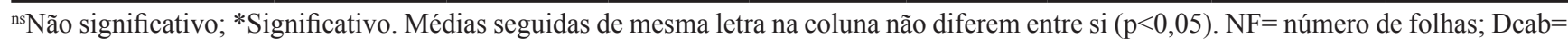
diâmetro da cabeça, Dcau= diâmetro do caule; $\mathrm{DH}=$ diâmetro horizontal; $\mathrm{MFC}=$ massa fresca da cabeça; $\mathrm{MSC}=$ massa seca da cabeça; Prod $=$ produtividade $\left({ }^{n n}\right.$ not significant; *significant. Average values followed by the same letter in the column do not differ $(\mathrm{p}<0.05)$. NF $=$ number of leaves; Dcab= head diameter, Dcau= stem diameter, $\mathrm{DH}=$ horizontal diameter; $\mathrm{MFC}=$ head fresh weight, $\mathrm{MSC}=$ head dry weight, Prod = productivity)

Tabela 3. Características agronômicas do repolho cultivado sobre resíduos de coberturas do solo (agronomic characteristics of cabbage grown on diferente plant residues used as soil mulching). Uberaba, IFTM, 2013.

\begin{tabular}{|c|c|c|c|c|c|c|c|c|}
\hline \multirow{2}{*}{ Coberturas } & \multirow{2}{*}{ NF } & Altura & Dcab & Dcau & DH & \multirow{2}{*}{$\begin{array}{c}\text { MFC } \\
\text { (kg) }\end{array}$} & \multirow{2}{*}{$\begin{array}{c}\text { MSC } \\
\text { (g) }\end{array}$} & \multirow{2}{*}{$\begin{array}{l}\text { Prod } \\
\text { (t/ha) }\end{array}$} \\
\hline & & \multicolumn{2}{|c|}{$(\mathrm{cm})$} & \multicolumn{2}{|c|}{$(\mathrm{mm})$} & & & \\
\hline Braquiária & $18 \mathrm{a}$ & $63,8 \mathrm{a}$ & $194,8 \mathrm{a}$ & $49,2 \mathrm{a}$ & $14,8 \mathrm{a}$ & $2,8 \mathrm{a}$ & $0,65 \mathrm{a}$ & $19,2 \mathrm{a}$ \\
\hline Crotalária & $16 \mathrm{c}$ & $60,9 \mathrm{a}$ & $179,7 \mathrm{c}$ & $41,5 \mathrm{~b}$ & $12,8 \mathrm{c}$ & $2,1 \mathrm{~b}$ & $0,66 \mathrm{a}$ & $14,7 \mathrm{~b}$ \\
\hline Milheto & $17 \mathrm{~b}$ & $64,6 \mathrm{a}$ & $190,8 \mathrm{~b}$ & $41,9 \mathrm{~b}$ & $13,7 \mathrm{~b}$ & $2,4 \mathrm{~b}$ & $0,53 \mathrm{~b}$ & $16,8 \mathrm{~b}$ \\
\hline Pousio & $17 \mathrm{~b}$ & $61,2 \mathrm{a}$ & $182,1 \mathrm{c}$ & $41,7 \mathrm{~b}$ & $13,7 \mathrm{~b}$ & $2,1 \mathrm{~b}$ & $0,46 \mathrm{~b}$ & $14,8 \mathrm{~b}$ \\
\hline $\mathrm{F}$ & $3,188^{*}$ & $2,508^{\text {ns }}$ & $2,659^{*}$ & $1,920^{*}$ & $2,230^{*}$ & $2,265^{*}$ & $2,203^{*}$ & $2,263 *$ \\
\hline CV (\%) & 4,89 & 3,20 & 4,04 & 10,75 & 6,85 & 14,91 & 19,78 & 14,92 \\
\hline DMS & 0,57 & 3,78 & 3,21 & 5,82 & 1,77 & 0,16 & 0,11 & 2,20 \\
\hline
\end{tabular}

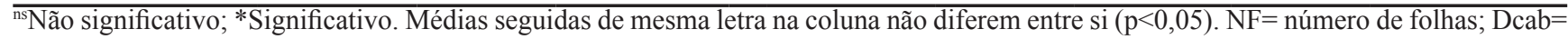
diâmetro da cabeça, Dcau= diâmetro do caule; $\mathrm{DH}=$ diâmetro horizontal; $\mathrm{MFC}=$ massa fresca da cabeça; $\mathrm{MSC}=$ massa seca da cabeça; Prod= produtividade $\left({ }^{\mathrm{ns}}\right.$ not significant, ${ }^{*}$ significant. Means followed by same letter in the column do not differ $(\mathrm{p}<0.05)$. NF $=$ number of leaves; $\mathrm{Dcab}=$ head diameter, $\mathrm{DCAU}=$ stem diameter; $\mathrm{DH}=$ horizontal diameter; $\mathrm{MFC}=$ head fresh weight, dry weight $\mathrm{MSC}=$ head dry weight; Prod = productivity).

\section{(Tabela 3).}

A absorção dos nutrientes pelas hortaliças, advindos da mineralização da matéria orgânica, depende da sincronia entre a decomposição e mineralização dos resíduos e a época de maior exigência nutricional da cultura (Fontanétti et al., 2006). A maior taxa de absorção e acúmulo de nutrientes pelo repolho ocorre entre 60 e 70 dias após o transplante, o que não ocorreu para a braquiária, a qual decompõe e libera os nutrientes contidos nos resíduos mais rapidamente, até 42 dias após o manejo. Apesar disso, os valores de massa fresca $(2,8 \mathrm{~kg})$ por cabeça obtidos nesse estudo para repolho sobre palhada de crotalária foram superiores a $1,3 \mathrm{~kg}$ relatados por Oliveira et al. (2005), 1,4 kg por Fontanétti et al. (2006) e 2,3 kg por Vargas et al. (2011) para essa cultura e cobertura.

Os valores de massa fresca de repolho obtidos nesse estudo foram superiores aos padrões exigidos pelo mercado consumidor brasileiro ( 1,0 a $1,5 \mathrm{~kg}$ de massa fresca comercial), mostrado por Lêdo et al.(2000). Isso pode ser justificado pelo maior período de cultivo, pois as colheitas tiveram início aos 100 dias após a semeadura.

O melhor desempenho agronômico da couve-flor e do repolho ocorreu quando a planta foi cultivada sobre os resíduos de braquiária. A produtividade do repolho também foi maior sobre os resíduos de braquiária, sendo os valores de massa fresca comercial da cultura superiores aos padrões exigidos pelo mercado consumidor brasileiro, que varia entre 1,0 e 1,5 $\mathrm{kg}$ (Silva et al., 2012).
A utilização das diferentes coberturas do solo e o plantio das mudas sobre os seus resíduos em decomposição é promissora no cultivo das hortaliças, pois além de melhorar os indicadores agronômicos comprovados neste estudo, a manutenção dos resíduos sobre o solo mantem a umidade por um período mais longo, diminuindo o custo da irrigação, minimizando a quantidade de adubo mineral devido à ciclagem de nutrientes, além de trazer outros benefícios, tais como controle da erosão e de algumas plantas invasoras.

\section{AGRADECIMENTOS}

Os autores agradecem o Instituto Federal do Triângulo Mineiro câmpus 
Uberaba pela infraestrutura disponibilizada, à Fapemig e ao CNPq pela concessão de bolsa de Iniciação Científica aos estudantes e à Fundação Agrisus pelo financiamento do projeto.

\section{REFERÊNCIAS}

ASSIS RL; OLIVEIRA CAO; PERIN A; SIMON GA; SOUZA JUNIOR BA. 2013. Produção de biomassa, acúmulo de nitrogênio por plantas de cobertura e efeito na produtividade do milho safrinha. Enciclopédia Biosfera, Centro Cientifico Conhecer 9: 1769-1775.

BOER CA; ASSIS RL; SILVA GP; BRAZ AJB; BARROSO ALL; CARGNELUTTI FILHO A; PIRES FR. 2008. Biomassa, decomposição e cobertura do solo ocasionada por resíduos culturais de três espécies vegetais na região Centro-oeste do Brasil. Revista Brasileira de Ciência do Solo 32 :843-851.

BRITO SS; SANTOS AC. 2010. Decomposição e mineralização de nutrientes em função da aplicação de diferentes fontes de matéria orgânica. Enciclopédia biosfera 6: 1-8.

CARVALHO AM; SOUZA LLP; GUIMARÃES JÚNIOR R; ALVES PCAC; VIVALDI LJ. 2011. Cover plants with potential use for crop-livestock integrated systems in the Cerrado region. Pesquisa Agropecuária Brasileira 46: 1200-1205.

CARVALHO C; KIST BB; POLL H. 2013. Anuário Brasileiro de Hortaliças. Santa Cruz do Sul: Editora Gazeta Santa Cruz, 88p.

CHIODEROLI CA; MELLO LMM; GRIGOLLI PJ; FURLANI CEA; SILVA JOR; CESARIN AL. 2012. Atributos físicos do solo e produtividade de soja em sistema de consórcio milho e braquiária. Revista Brasileira de Engenharia Agrícola Ambiental 16: 37-43.

COMISSÃO DE FERTILIDADE DO SOLO DO ESTADO DE MINAS GERAIS. 1999. Recomendações para o uso de corretivos e fertilizantes em Minas Gerais: $5^{a}$ aproximação. Viçosa: CFSEMG, 359 p.

CRUSCIOL CAC; SORATTO RP. 2009. Nitrogen supply for cover crops and effects on peanut grown in succession under a no-till system. Agronomy Journal 101: 40-46.

DINIZ ER; SANTOS RHS; URQUIAGA SS; PETERNELLI LA; BARRELLA
TP; FREITAS GB. 2007. Green manure incorporation timing for organically grown broccoli. Pesquisa Agropecuária Brasileira 42: 199-206.

EMBRAPA, Centro Nacional de Pesquisa de solos. 2006. Sistema brasileiro de classificação de solos. 2a ed. RJ: Embrapa. 306p.

ESPINDOLA JAA; GUERRA JGM; ALMEIDA DL; TEIXEIRA MG; URQUIAGA S. 2006. Decomposição e liberação de nutrientes acumulados em leguminosas herbáceas perenes consorciadas com bananeira. Revista Brasileira de Ciência do Solo 30: 321-328.

FABIAN AJ. 2009. Plantas de cobertura: efeito nos atributos do solo e na produtividade de milho e soja em rotação. Jaboticabal: UNESPFCAV. 83p. (Tese doutorado).

FONTANÉTTI A; CARVALHO GJ; GOMES LAA; ALMEIDA K; MORAES SRG; TEIXEIRA CM. 2006. Adubação verde na produção orgânica de alface americana e repolho. Horticultura Brasileira 24: 146-150.

HORTIBRASIL, Instituto Brasileiro de Qualidade em Horticultura. Normas de Identidade, Padronização e Classificação da Couve-flor. 2011. Disponível em: <http://www.hortibrasil. org.br/classificacao/couve-flor/couve-flor. html $>$. Acessado em 26/02/2014.

LÊDO FJS; SOUZA JA; SILVA MR. 2000. Avaliação de cultivares e híbridos de repolho no Estado do Acre. Horticultura Brasileira 18: 138-140.

LEITE LFC; FREITAS RCA; SAGRILO E; GALVÃO SRS. 2010. Decomposição e liberação de nutrientes de resíduos vegetais depositados sobre Latossolo Amarelo no Cerrado Maranhense. Revista Ciência Agronômica 41: 29-35.

MAROUELLI WA; ABDALLA RP; MADEIRA NR; OLIVEIRA AS; SOUZA RF. 2010. Eficiência de uso de água e produção do repolho sobre diferentes quantidades de palha em plantio direto. Pesquisa Agropecuária Brasileira 45: 369-375.

MONTEIRO BCBA; CHARLO HCO; BRAZ LT. 2010. Desempenho de híbridos de couveflor de verão em Jaboticabal. Horticultura Brasileira 28: 115-119.

MORAIS JUNIOR PO; CARDOSO AF; LEÃO EF; PEIXOTO N. 2012. Desempenho de cultivares de couve-flor de verão em Ipameri. Ciência Rural 42: 1923-1928.

OLIVEIRAFL; RIBAS RGT; JUNQUEIRA RM;
PADOVAM MP; GUERRA JGM; ALMEIDA DL; RIBEIRO RLD. 2005. Desempenho do consórcio entre repolho e rabanete com précultivo de crotalária, sob manejo orgânico. Horticultura Brasileira 23: 184-188.

PACHECO LP; LEANDRO WM; MACHADO PLOA; ASSIS RL; COBUCCI T; MADARI BE; PETTER FA. 2011. Produção de fitomassa e acúmulo e liberação de nutrientes por plantas de cobertura na safrinha. Pesquisa Agropecuária Brasileira 46: 17-25.

RICCI MSF; CASALI VW; CARDOSO AA; RUIZ HA. 1995. Teores de nutrientes em duas cultivares de alfaces adubadas com composto orgânico. Pesquisa Agropecuária Brasileira 30: 1035-1039.

SILVA KS; SANTOS ECM; BENETT CGS; LARANJEIRA LT; EBERHARDT NETO E; COSTA E. 2012. Produtividade e desenvolvimento de cultivares de repolho em função de doses de boro. Horticultura Brasileira 30: 520-525.

TEIXEIRA CM; LOSS A, PEREIRA MG; PIMENTEL C. 2012. Decomposição e ciclagem de nutrientes dos resíduos de quatro plantas de cobertura do solo. Idesia 30: 55-64.

TORRES JLR; PEREIRA MG. 2008. Dinâmica do potássio nos resíduos vegetais de plantas de cobertura no Cerrado. Revista Brasileira de Ciência do Solo 32:1609-1618.

TORRES JLR; PEREIRA MG; ANDRIOLI I; FABIAN AJ; POLIDORO JC. 2005. Decomposição e liberação de nitrogênio de resíduos culturais de plantas de cobertura em um solo de Cerrado. Revista Brasileira de Ciência do Solo 4: 609-618.

TORRES JLR; PEREIRA MG; FABIAN AJ. 2008. Produção de fitomassa por plantas de cobertura e mineralização de seus resíduos em plantio direto. Pesquisa Agropecuária Brasileira 43: 421-428.

UBERABA EM DADOS, Prefeitura Municipal de Uberaba. Edição 2009, 23 p. Disponível em: <http:www.uberaba.mg.gov.br/portal/ acervo/desenvolvimento/arquivos/uberaba em_dados/Edicao_2009/Capitulo01.pdf $>$. Acesso em 21.02.2014.

VARGAS TO; DINIZ ER; SANTOS RHS; LIMA CTA; URQUIAGA S; CECON PR. 2011. Influência da biomassa de leguminosas sobre a produção de repolho em dois cultivos consecutivos. Horticultura Brasileira 29: $562-568$ 